\title{
Autologous hematopoietic stem cell transplantation in autoimmune diseases - a brand new standard. Where do we go from here?
}

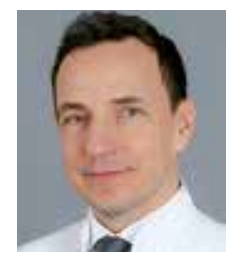

\section{Emilian Snarski}

Department and Clinic of Hematology, Oncology and Internal Medicine, Warsaw Medical University, Poland

For many years autologous hematopoietic stem cell transplantation (AHSCT) has been viewed as experimental treatment of severe autoimmune diseases. However, as randomized trials have finished we are now facing a new reality - autologous hematopoietic stem cell transplantation has been acknowledged by major medical societies as standard treatment of severe autoimmune diseases [1-3].

How does AHSCT work in autoimmune diseases? First the hematopoietic stem cells are collected from the patient. Second conditioning is given - a strong chemotherapy that can eradicate the underlying autoimmune disease. How strong is this chemotherapy? To put it into perspective: the systemic sclerosis patient who gets 1 gram of cyclophosphamide in monthly infusions if transplanted will get 24 grams of cyclophosphamide within 4 days together with additional antithymocyte globulin - and this is one of the least intensive approaches that we use in hematopoietic stem cell transplantation. Third step - the infusion of hematopoietic stem cells. The therapeutic effect is achieved by chemotherapy; the stem cells just help to regenerate the hematopoietic system. The exact mechanism of action is still not fully understood - is it the restart or reset of immune responses or is it a shift towards a more tolerant immune system? More research is needed to answer this question.

So where are we now with this approach? There two autoimmune disease in which AHSCT has just been recognized as standard. The first disease in which AHSCT has reached the status of standard is systemic sclerosis [1]. EULAR was first to acknowledge that there are enough data on AHSCT to set it as standard for severe cases of systemic sclerosis. Rheumatologists were the first and so far the only society other than hematologists to acknowledge that AHSCT works. The second disease is multiple sclerosis. The European Society for Blood and Marrow Transplantation and the American Society for Blood and Marrow Transplantation have just named AHSCT as the standard treatment of relapsing remitting MS on the basis of a few randomized trials and multiple one arm trials [2, 3].

What is the situation with other autoimmune diseases? AHSCT can be a clinical option in other autoimmune diseases - especially in refractory cases of systemic lupus, Crohn's disease, chronic demyelinating polyneuropathy, neuromyelitis optica and other autoimmune conditions [3]. It is very unlikely that we will see many new randomized clinical trials in those diseases in coming years. However, even one arm single center studies show that AHSCT can achieve durable remissions where other immunosuppressive or immunomodulatory treatments failed $[4,5]$.

What is the situation in Poland? The situation in Poland is very unique - all transplantation procedures are paid by the public payer and the indications are made by the center. This opens the possibility to apply AHSCT for any patient with any severe autoimmune disease - the only requirement is combined qualification by a rheumatologist or neurologist and hematologist. The transplantation centers in Katowice and Warsaw have already transplanted patients with multiple sclerosis, systemic sclerosis and type 1 diabetes [6-8]. There are also other centers that are willing to accept patients.

What are the limitations of AHSCT in autoimmune disease? The current AHSCT protocols target the inflammation well. Thus the more inflammation we see the more response we will get after AHSCT. What the current AHSCT does not target so efficiently are the long living plasma cells. This creates problems especially in autoimmune disease with antibody production. Understanding how to destroy this subpopulation of cells may contribute 
to the increase in the effectiveness of this approach. Another problem that needs to be solved is qualification of patients. The transplant outcomes rely on appropriate qualification of patients. Finding the right time point and efficient conditioning for AHSCT seem to be the current challenges.

What are the dangers and how to overcome them? The risks of severe complications and mortality are low in current AHSCT protocols in autoimmune diseases. Currently mortality is very close to zero. This has been achieved by accumulating experience, proper patient selection and reduction of toxicity of conditioning. We also have to remember that patients themselves are willing to accept much higher risks than are connected with AHSCT - especially as the disease progresses [9].

Where do we go from here? There are many areas of improvement. The first concerns the drugs used for conditioning - there is a possibility of delivering more efficient yet less toxic chemotherapies prior to AHSCT. We do not know what the optimal pre- and post-AHSCT treatment is. The effect of transplantation might be life-saving and all patients can generally feel better after AHSCT but not all return to the full pre-disease activity [10] - improving the proportion of patients who return to full activity is another goal to be achieved. AHSCT can also let us finally answer how it suppresses the diseases - maybe we could replicate this effect either by drugs or by delivery of modified immune cells?

Summarizing, AHSCT for autoimmune diseases is not a magic bullet, but it is a bullet that can hit the disease hard and induce long lasting remissions. We are entering a new era for AHSCT in autoimmune diseases - the new standard in severe and refractory cases.

The author declares no conflict of interest.

\section{References}

1. Kowal-Bielecka O, Fransen J, Avouac J, et al. Update of EULAR recommendations for the treatment of systemic sclerosis. Ann Rheum Dis 2017; 76: 1327-1339.

2. Cohen JA, Baldassari LE, Atkins HL, et al. Autologous Hematopoietic Cell Transplantation for Treatment-Refractory Relapsing Multiple Sclerosis: Position Statement from the American Society for Blood and Marrow Transplantation. Biol Blood Marrow Transplant 2019; 25: 845-854.

3. Sharrack B, Saccardi R, Alexander T, et al. Autologous haematopoietic stem cell transplantation and other cellular therapy in multiple sclerosis and immune-mediated neurological diseases: updated guidelines and recommendations from the EBMT Autoimmune Diseases Working Party (ADWP) and the Joint Accreditation Committee of EBMT and ISCT (JACIE). Bone Marrow Transplant 2019. doi:10.1038/s41409-019-0684-0 [Epub ahead of print].

4. Burt RK, Craig RM, Milanetti F, et al. Autologous nonmyeloablative hematopoietic stem cell transplantation in patients with severe antiTNF refractory Crohn disease: long-term follow-up. Blood 2010; 116: 6123-6132.

5. Burt RK, Han X, Gozdziak P, et al. Five year follow-up after autologous peripheral blood hematopoietic stem cell transplantation for refractory, chronic, corticosteroid- dependent systemic lupus erythematosus : effect of conditioning regimen on outcome. Bone Marrow Transplant 2018; 53: 692-700.

6. Szczechowski L, Śmiłowski M, Helbig G, et al. Autologous hematopoietic stem cell transplantation (AHSCT) for aggressive multiple sclerosis - whom, when and how. Int J Neurosci 2016; 126: 867-871.

7. Kyrcz-Krzemien S, Helbig G, Torba K, et al. Safety and efficacy of hematopoietic stem cells mobilization in patients with multiple sclerosis. Hematology 2016; 21: 42-45.

8. Snarski E, Milczarczyk A, Torosian T, et al. Independence of exogenous insulin following immunoablation and stem cell reconstitution in newly diagnosed diabetes type I. Bone Marrow Transplant 2011; 46: 562-566.

9. Chacińska W, Brzostowska M, Nojszewska M, et al. "Cure" for multiple sclerosis (MS) - Evolving views of therapy goals in patients on different stages of the disease: A pilot study in a cohort of Polish MS patients. Brain Behav 2017; 7: e00701.

10. Atkins HL, Bowman M, Allan D, et al. Immunoablation and autologous haemopoietic stem-cell transplantation for aggressive multiple sclerosis: a multicentre single-group phase 2 trial. Lancet 2016; 388: 576-585. 